\title{
APPLICATION DE LA THÉORIE DES PROCESSUS ALÉATOIRES A L'ESTIMATION DE LA PRÉCISION D'UN INVENTAIRE FORESTIER PAR ÉCHANTILLONNAGE SYSTÉMATIQUE
}

\author{
X. GIUDICELLI, J.-P. LANLY*, J.-B. OUAKAM et M. PIETRI \\ Institut de Statistique de l'Université de Paris \\ * Centre technique forestier tropical, \\ 94 - Nogent-sur-Marne
}

\begin{abstract}
RÉSUMÉ
L'estimation de l'erreur statistique dans les dispositifs de sondage systématiques, largement utilisés en foresterie, est un problème qui n'est pas soluble dans le strict cadre de la théorie des sondages. Conformément à une suggestion faite par Cochran et d'autres, les auteurs proposent une solution en considérant la forêt étudiée comme une réalisation d'un processus aléatoire stationnaire et isotrope. Cette méthode permet une estimation de la variance de la moyenne de l'échantillon systématique en fonction de la corrélation moyenne entre deux unités d'échantillonnage de la population étudiée et celle entre deux unités de l'échantillon. L'estimation ainsi calculée dépend donc de la géométrie de l'échantillon et de la forme du corrélogramme représentant la variation du coefficient de corrélation entre les valeurs d'un paramètre étudié dans deux unités en fonction de la distance qui les sépare. Des procédés de calcul sont donnés et une application de la méthode est faite utilisant les résultats d'un inventaire complet en forêt tropicale. La méthode apparaît d'autant plus satisfaisante que la maille du réseau des unités de sondage est plus rectangulaire et que le paramètre étudié a une distribution plus « contagieuse».
\end{abstract}

\section{INTRODUCTION}

Étant donné un peuplement forestier, considéré comme surface de référence - parcelle de forêt, série, forêt, zone forestière - l'objectif de l'inventaire par sondage de ce peuplement est d'obtenir au moindre coût pour une précision fixée à l'avance (1) l'estimation d'un ou de plusieurs paramètres caractérisant ce peuplement et dont la connaissance est utile pour sa gestion et sa mise en valeur. L'estimation cherchée peut être une valeur moyenne du paramètre à l'unité de surface ou le total de ce paramètre sur l'ensemble du peuplement. Les paramètres considérés sont soit des nombres de tiges d'une essence ou d'un groupe d'es-

(1) Ou avec la meilleure précision en fonction d'un coût fixé a priori. 
sences donné, par catégorie de grosseur ou toutes catégories au-dessus d'un diamètre minimum, soit les volumes correspondants. Si l'on ne tient pas compte des erreurs dues à l'application de tarifs de cubage, au rangement des arbres par catégorie de grosseur ou aux erreurs d'observations inévitables, la précision du sondage est caractérisée par la variance de l'estimation fournie par l'échantillon adopté.

La nature du dispositif de sondage adopté sera fonction d'une part de la précision que l'on se fixe à l'avance sur un paramètre, et, d'autre part, de la nature, de l'importance et aussi de l'accessibilité $\left({ }^{1}\right)$ du peuplement forestier à inventorier. Celui-ci sera considéré soit comme une population d'arbres, notamment dans le cas de certaines plantations artificielles, soit comme une population d'éléments de surface jointifs, de taille égale de préférence. Les unités d'échantillonnage sont donc, suivant les cas, des arbres ou des éléments de surface. Dans ce deuxième cas, le sondage sera qualifié d'aréolaire. Le sondage pourra être à un seul degré - les unités d'échantillonnage provenant du découpage du peuplement à inventorier n'étant pas considérées elles-mêmes comme des populations d'unités plus petites — ou à plusieurs degrés, les unités tirées à un degré étant elle-mêmes sondées lors du degré suivant du sondage. A chaque degré du sondage, le tirage de chacune des unités d'échantillonnage peut être fait au hasard - par un des nombreux procédés d'échantillonnage aléatoire possibles - ou d'une manière systématique (prélèvement d'une fiche toutes les $k$ fiches d'un fichier, ou éléments de surface de l'échantillon répartis suivant une grille à maille constante placée sur la surface à inventorier, etc., seule la première unité étant tirée au hasard).

En matière de peuplements forestiers, les dispositifs de sondage de type systématique offrent de nombreux avantages pratiques. Aussi sont-ils préférés à des dispositifs aléatoires qui sont plus difficiles à mettre en œuvre et plus coûteux. L'adoption des dispositifs systématiques se heurte essentiellement au problème difficile à résoudre dans la majorité des cas, de la détermination d'une estimation de la variance de l'estimation fournie. La méthode exposée ci-dessous apporte une solution à ce problème important. Elle a été mise au point pour des dispositifs systématiques aréolaires à un degré. Sa transposition reste à faire sur les sondages non aréolaires et dans les dispositifs à plusieurs degrés.

Dans un premier chapitre, nous développerons les données du problème et les solutions approchées imaginées par les statisticiens dans le cadre de la théorie des sondages. Dans une deuxième partie seront exposés le «modèle » adopté et les calculs permettant la détermination d'une estimation de la variance. Enfin, l'ensemble de cette méthode sera appliquée aux données d'un inventaire exhaustif d'un massif de forêt dense tropicale au Cameroun et les résultats commentés. Sauf mention particulière, on supposera implicitement dans le reste de l'article qu'il s'agit de dispositifs systématiques aréolaires à un degré.

\section{I. - POSITION DU PROBLÈME}

\section{1. - Avantages des dispositifs systématiques}

1.11. Si l'on fait abstraction de l'inconvénient majeur du dispositif systématique, à savoir la difficulté d'estimer la variance de l'estimateur, celui-ci apparaît particulièrement intéressant.

(1) Et plus généralement des différents facteurs logistiques qui influent sur le coût de l'opération, l'importance de ceux-ci dans le choix d'un dispositif de sondage étant accrue dans les inventaires forestiers tropicaux. 
Il permet, tout d'abord, d'obtenir un estimateur sans biais du paramètre. Cette propriété de l'estimateur fourni n'est pas en contradiction avec l'impossibilité où l'on se trouve d'estimer la variance de cet estimateur. En effet $\mathrm{N}$ étant le nombre total d'unités de la population, $n$ celui de l'échantillon, il y a $k=\frac{N}{n}$ échantillons de $n$ unités dont la réunion constitue la population totale. Chacun de ces $k$ échantillons peut être assimilé à une grappe et le tirage au sort de la première unité d'un échantillon systématique équivaut au tirage au sort d'une grappe parmi les $k$ grappes de la population. On comprend bien ici pourquoi, bien que l'on ait affaire à un échantillonnage aléatoire, l'estimation de la variance n'est pas théoriquement possible : en effet, si on peut avoir une estimation de la variance «intra » à l'intérieur d'une grappe quelconque, on ne peut par contre déterminer la composante «inter-grappes » de la variance.

1.12. Le dispositif systématique apparaît - toutes choses égales d'ailleurs - comme un dispositif particulièrement efficace pour de nombreuses raisons que nous énumérons rapipement ci-dessous.

1.121. Le dispositif systématique est plus simple à mettre en œuvre sur le terrain qu'un échantillonnage aléatoire de même importance. Qu'il s'agisse de bandes continues régulièrement espacées ou de lignes équidistantes d'unités d'échantillonnage à intervalle constant sur ces lignes, et quelle que soit la forme de la maille du réseau de celles-ci, le repérage au sol des unités est simple : en effet, seule l'implantation de la première unité pose un problème de repérage, l'emplacement des autres unités se déduisant de celui de la première par des orientements et des distances déterminés une fois pour toutes. Cette considération prend toute son importance dans les inventaires de certaines forêts denses tropicales où la rareté des points de repère facilement accessibles rendrait le positionnement d'unités implantées au hasard particulièrement difficile.

1.122. Le dispositif systématique est par suite plus rapide à mettre en œuvre. Non seulement le tirage de l'échantillon prend moins de temps puisqu'il se limite au tirage d'une seule unité de l'échantillon, mais encore son implantation s'exécute plus rapidement, le cheminement à suivre d'une unité à la suivante étant toujours le même.

1.123. Une caractéristique du dispositif systématique sur laquelle il apparaît nécessaire d'insister est sa plus grande sécurité sur le plan opérationnel. En effet, il constitue un cadre très étroit dont l'exécutant n'est pas tenté de sortir, ce qui n'est pas le cas d'un dispositif strictement aléatoire où, certaines fois, l'observateur peut être enclin à choisir, en modifiant, ne serait-ce que légèrement, les emplacements des unités d'échantillonnage pour plus de commodité.

1.124. Sa simplicité de mise en cuvre fait que, à caractéristiques de sondage identiques - notamment nombre, forme et dimensions des unités d'échantillonnage - le dispositif systématique est finalement moins coûteux qu'un dispositif aléatoire. Ceci est particulièrement vrai dans les inventaires de forêt dense tropicale où une composante importante du coût est le prix de revient des ouvertures du sous-bois pour permettre le cheminement à l'intérieur du massif forestier : l'alignement des unités d'échantillonnage permet une réduction notable du coût des inventaires dans ce type de forêt.

1.125. Un dernier avantage, non des moindres, du dispositif systématique, est la répartition régulière sur le peuplement inventorié des informations fournies. Relativement 
à un paramètre donné on peut ainsi découper le massif inventorié en différentes zones correspondant chacune à une classe de valeur du paramètre et comprenant les « surfaces d'extension » des unités d'échantillonnage caractérisées par cette valeur. Bien qu'un tel découpage cartographique soit nécessairement simpliste, il n'en demeure pas moins qu'il peut être utile à plus d'un titre et qu'il est grandement facilité par la répartition systématique des unités d'échantillonnage.

\section{2. - Inconvénient des dispositifs systématiques}

Le principal inconvénient des dispositifs systématiques étant l'impossibilité d'obtenir une estimation de la variance de la moyenne de l'échantillon, les statisticiens ont adopté suivant les cas des méthodes approchées d'estimation. Précisons toutefois que cet inconvénient n'existe pas pour des paramètres dont la répartition des valeurs sur l'ensemble des unités de la population est distribuée « au hasard $\left(^{1}\right)$ : pour ces paramètres la variance est donnée par la formule du sondage aléatoire. Malheureusement les paramètres dendrométriques mesurés dans les forêts aménagées et même dans les forêts vierges relativement à une majorité d'essences ont des distributions le plus souvent contagieuses qui n'ont rien à voir avec une distribution « au hasard ». Néanmoins par commodité les inventoristes ont tendance à utiliser dans tous les cas et quel que soit le paramètre recherché, les formules des sondages strictement aléatoires alors que l'extrapolation de celles-ci aux dispositifs systématiques doit être, comme nous venons de le dire, limitée à un certain type de paramètres, non influencée en particulier par les phénomènes de contagion.

1.21. La méthode préconisée le plus souvent consiste à découper arbitrairement la surface inventoriée en strates de taille égale contenant chacune un même nombre d'unités d'échantillonnage.

On aboutit aux formules suivantes (avec les notations habituelles) :

$$
\text { a) } s^{2}(\bar{x})=\frac{1-f}{n} \frac{1}{n-1} \sum_{\mathrm{j}=1}^{n-1} \frac{\left(x_{j+1}-x_{j}\right)^{2}}{2}
$$

(DESABIE : $n-1$ strates de 2 unités successives $j$ et $j+1$ )

$$
\text { b) } \left.s^{2}(x)=\frac{1-f}{n} \frac{1}{3 n^{\prime}} \llbracket \sum_{i=1}^{n} x_{i}^{2}-4 \sum_{j=1}^{n \prime} \bar{x}_{j}^{2}\right\rceil
$$

(YATES : $n^{\prime}$ strates contenant chacune 4 unités aux quatre coins de la maille du réseau des unités,

$\sum_{j=i}^{n^{\prime}} \bar{x}_{j}{ }^{2}$ étant la somme des carrés des moyennes par strate)

1.22. Cochran propose dans le cas d'un sondage à une dimension avec une variation

(') C'est-à-dire que l'espérance mathématique de la moyenne en chaque unité de la strate est constante. 
linéaire du paramètre le long de la ligne d'unités d'échantillonnage d'utiliser la formule suivante :

$$
s^{2}(x)=\frac{1-f}{n} \frac{\sum_{i}\left(x_{i}-2 x_{i+k}+x_{i+2 k}\right)^{2}}{6(n-2)}
$$

où $i+k$ et $i+2 k$ représentent les indices des unités consécutives de l'unité $i$ dans l'échantillon systématique.

1.23. Des formules plus complexes ont été mises au point par différents spécialistes des sondages. La plus connue est celle adoptée par YATES des « différences compensées 》 portant sur une combinaison linéaire des valeurs du paramètre sur 9 unités d'échantillonnage consécutives, de la forme :

$$
d=\frac{1}{2} x_{1}-x_{2}+x_{3}-x_{4}+x_{5}-x_{6}+x_{7}-x_{8}+\frac{1}{2} x_{9}
$$

La variance est alors donnée par la formule :

$$
s^{2}(x)=\frac{1-f}{n} \sum_{u=1}^{g} \frac{d u^{2}}{7,5}
$$

où $g$ désigne le nombre de différences $d_{u}, 7,5$ représentant la somme des carrés des coefficients des 9 valeurs entrant dans une différence $d_{u}$.

1.231. En définitive, il apparaît nettement que malgré les nombreux avantages d'ordre pratique des dispositifs systématiques, on se heurte dans l'estimation de la variance de la moyenne donnée par l'échantillon à un problème impossible à résoudre d'une manière rigoureuse dans le strict cadre de la théorie des sondages, et que les spécialistes y ont remédié par des « expédients » plus ou moins adaptés suivant le type de distribution des paramètres recherchés dans les populations sondées. Cet état de choses a conduit certains de ces spécialistes, notamment Cochran, à recommander l'utilisation de la théorie des processus aléatoires pour résoudre le problème de l'estimation de la variance dans les dispositifs systématiques.

\section{II. - MÉTHODE PROPOSÉE}

\section{1. - Hypothèses probabilistes}

2.11 - Après avoir dressé la carte de la forêt étudiée, on peut considérer celle-ci comme une portion de plan. Pour faciliter les observations, on ne prend pas comme unités statistiques les arbres, mais des parcelles carrées de même taille obtenues par quadrillage de la portion de plan représentant la forêt, et dont le côté sert d'unité de longueur. La forêt est alors l'ensemble de ces parcelles qui sont repérées par les coordonnées entières de leur centre par rapport à un système d'axes rectangulaires convenablement choisis.

$\mathrm{Si} \mathrm{Z}_{i j}$ est une caractéristique numérique des arbres de la placette de coordonnées $(i, j)$ (comme, par exemple, le nombre d'arbres d'une classe de diamètre et d'une essence donnée observé sur la placette $(i, j)$, ou le volume correspondant observé sur la même placette), il est naturel de considérer $Z_{i j}$ comme une variable aléatoire en ce sens que $Z_{i j}$ peut 
prendre un ensemble de valeurs numériques sans qu'il soit possible de dégager les conditions qui détermineraient d'une façon certaine la valeur prise réellement par $\mathbf{Z}_{i j}$.

Dans cette optique, l'ensemble des variables aléatoires $Z_{i j}$ que l'on peut noter :

$$
Z_{i j} ;(i, j) \varepsilon \mathbf{I} \times \mathbf{J}
$$

(si I et $\mathbf{J}$ sont les ensembles des valeurs de $i$ et de $j$ respectivement) s'appelle un processus aléatoire dans $\mathrm{R}^{2}$ et l'ensemble des valeurs prises réellement par les $Z_{i j}$ forme une réalisation de ce processus aléatoire.

L'intérêt de cette construction, somme toute assez naturelle, est double :

- elle permet de formaliser certaines propriétés de la forêt en termes probabilistes comme, par exemple, le lien existant entre les nombres d'arbres d'une essence donnée dans deux placettes différentes, que l'on peut exprimer par une fonction de corrélation;

— elle permet d'évaluer la précision d'un sondage systématique.

\section{2. - Rappels sur les processus aléatoires}

2.21. Processus numérique du second ordre dans $\mathrm{R}^{2}$.

Si $t$ est un paramètre décrivant $\mathrm{R}^{2}$, c'est-à-dire un vecteur de $\mathrm{R}^{2}$, un processus aléatoire dans $\mathrm{R}^{2}$ est un ensemble de variables aléatoires réelles que nous noterons :

$$
\mathrm{Z}(t) ; t \varepsilon \mathrm{R}^{2} !
$$

Si quels que soient $t$ et $t^{\prime}$ dans $\mathrm{R}^{2}$ l'espérance mathématique $\mathrm{E}\left(\mathrm{Z}(t) \mathbf{Z}\left(t^{\prime}\right)\right)$ de $\mathrm{Z}(t) \mathbf{Z}\left(t^{\prime}\right)$ existe, on dit que le processus est du second ordre.

On démontre que si un processus aléatoire est du second ordre :

- chaque variable aléatoire $Z(t)$ possède une espérance mathématique

$$
m(t)=\mathrm{E}\lceil\mathrm{Z}(t)]
$$

- chaque variable aléatoire $Z(t)$ possède une variance

$$
\mathrm{V}(t)=\mathrm{E}|\mathrm{Z}(t)-m(t)|^{2}
$$

— on peut définir la fonction de covariance sur $\mathrm{R}^{2} \times \mathrm{R}^{2}$ à valeurs dans $\mathrm{R}$ :

$$
\mathrm{C}\left(t, t^{\prime}\right)=\mathrm{E}\left|(\mathrm{Z}(t)-m(t))\left(\mathrm{Z}\left(t^{\prime}\right)-m\left(t^{\prime}\right)\right)\right|
$$

et on a : $\mathrm{V}(t)=\mathrm{C}(t, t)$

— on peut définir la fonction de corrélation sur $\mathrm{R}^{2} \times \mathrm{R}^{2}$ à valeurs dans $\mathrm{R}$ :

$$
\rho\left(t, t^{\prime}\right)=\frac{\mathrm{C}\left(t, t^{\prime}\right)}{\sqrt{\mathrm{C}(t, t) \mathrm{C}\left(t^{\prime}, t^{\prime}\right)}}
$$

\subsection{Processus stationnaires du second ordre.}

Un processus du second ordre sur $\mathrm{R}^{2}$ est dit stationnaire si les moments du premier et du second ordre sont invariants par translation dans $\mathrm{R}^{2}$. 
Cela s'exprime ainsi :

$$
\begin{aligned}
& m(t)=m \text { quel que soit } t \varepsilon \mathrm{R}^{2}, \\
& \mathrm{C}\left(t, t^{\prime}\right)=\mathrm{C}\left(t^{\prime}-t\right) \text { quels que soient } t \varepsilon \mathrm{R}^{2} \text { et } t^{\prime} \varepsilon \mathrm{R}^{2} .
\end{aligned}
$$

Ceci entraîne :

$$
\begin{aligned}
& \mathrm{V}(t)=\mathrm{C}(t, t)=\mathrm{C}(t-t)=\sigma^{2} \text { quel que soit } t \varepsilon \mathrm{R}^{2} \\
& \text { et } \rho\left(t, t^{\prime}\right)=\frac{\mathrm{C}\left(t^{\prime}-t\right)}{\sigma^{2}}=\rho\left(t^{\prime}-t\right) .
\end{aligned}
$$

Par suite, $\rho$ n'est fonction que d'une variable $u$ décrivant $\mathbf{R}^{2}$.

On démontre que $\rho$ est une fonction définie sur $\mathrm{R}^{2}$ de type positif, c'est-à-dire que, quel que soit l'ensemble de vecteurs de $\mathrm{R}^{2}\left\{t_{1}, t_{2}, \ldots ., t_{n}\right\}$ et quel que soit l'ensemble de nombres réels $\left\{a_{1}, a_{2}, \ldots ., a_{n}\right\}$ on a :

$$
\sum_{i=1}^{n} \sum_{j=1}^{n} a_{i} a_{j} \rho\left(t_{i}-t_{j}\right) \geqslant 0 .
$$

On démontre encore que, si $\rho(u)$ est continue sur $\mathbf{R}^{2}$, il existe un vecteur aléatoire de $\mathbf{R}^{2}$ dont $\rho$ est la fonction caractéristique. La distribution de ce vecteur aléatoire est appelée distribution spectrale du processus.

\subsection{Processus stationnaires isotropes.}

Un processus stationnaire du second ordre est dit isotrope si sa fonction de corrélation est invariante par rotation dans $\mathbf{R}^{2}$. Cela se traduit par :

$$
\text { si } u=\left(\begin{array}{l}
u_{1} \\
u_{2}
\end{array}\right), \rho(u)=\rho\left(\sqrt{u_{1}^{2}+u_{2}^{2}}\right)
$$

n'est donc fonction que d'une variable réelle positive $d$.

On démontre alors que $\rho(d)$ est la fonction caractéristique d'une distribution spectrale radiale $\left({ }^{1}\right)$.

\section{Exemples :}

$1^{0}$ Si $\lambda>0, \rho(d)=e^{-\lambda d^{2}}$ est la fonction caractéristique de la distribution radiale qui a pour densité en coordonnées polaires

$$
f(r)=k r e^{-\frac{r^{2}}{4 \lambda}}
$$

où $k$ est une constante.

(1) Un vecteur de $\mathrm{R}^{2}$ suit une loi de distribution radiale si sa fonction de répartition au point $\left(x_{1}, x_{2}\right.$ ne dépend que de $\sqrt{x_{1}^{2}+x_{2}^{2}}$.

Il est à remarquer qu'en général $\rho(d)$ n'est pas une fonction de $d$ de type positif. 
$2^{0} \rho(d)=e^{- \text {ad }}(a>0)$ est la fonction de corrélation associée à la densité spectrale

$$
f(r)=\left(1+\frac{r^{2}}{a^{2}}\right)^{-\frac{3}{2}}
$$

$3^{0}$ On en déduit que la fonction suivante

$$
\rho(d)=p e^{-a d}+q e^{-b d} \quad(a>0, b>0, p+q=1)
$$

est aussi une fonction de corrélation d'un processus stationnaire isotrope.

\section{3. - Justification de l'utilisation d'un processus stationnaire isotrope du second ordre dans le problème posé}

Puisque l'on doit évaluer le nombre d'arbres d'une essence donnée, la variable aléatoire, liée à la placette $(i, j)$ que l'on considère est :

$\mathrm{X}_{\mathrm{ij}}=$ nombre d'arbres de l'essence donnée pouvant apparaître sur la placette $(i, j)$.

Nous supposerons que le processus : $\left[\mathrm{X}_{i j} ;(i, j) \varepsilon \mathrm{I} \times \mathbf{J}\right]$ est stationnaire au second ordre et isotrope, c'est-à-dire que, quels que soient $i \varepsilon \mathrm{I}, u \varepsilon \mathrm{I}, j \varepsilon \mathrm{J}, v \varepsilon \mathrm{J}$, on a :

$$
\begin{aligned}
& \mathrm{E}\left[\mathrm{X}_{i j}\right]=m \\
& \mathrm{E}\left[\left(\mathrm{X}_{i j}-m\right)^{2}\right]=\sigma^{2} \\
& \frac{\mathrm{E}\left|\left(\mathrm{X}_{i j}-\mathrm{m}\right)\left(\mathrm{X}_{u v}-m\right)\right|}{\sigma^{2}}=\rho(d)
\end{aligned}
$$

avec $d=\sqrt{(i-u)^{2}+(j-v)^{2}}$ qui est la distance géométrique entre les centres des placettes $(i, j)$ et $(u, v)$.

Dans le cas d'une forêt :

- l'hypothèse de stationnarité peut se justifier par une certaine stabilité dans toute la forêt des conditions d'apparition et de développement des arbres; s'il semble que cette stabilité n'est pas réalisée - par exemple lorsqu'une certaine tendance apparaît due à la modification de facteurs écologiques (relief, sol, microclimat) - l'hypothèse de stationnarité peut être acceptée au niveau de strates judicieusement choisies;

- l'hypothèse d'isotropie, vérifiée si la corrélation entre les valeurs de la caractéristique cherchée dans deux parcelles situées à une distance donnée est la même dans toutes les directions, n'est satisfaite dans une forêt que s'il n'apparaît pas une ou plusieurs directions privilégiées de la répartition de l'essence étudiée;

- il est également logique de supposer que le phénomène de liaison s'atténue lorsque la distance entre deux placettes augmente et qu'il devient négligeable à partir d'une certaine distance. Dans ces conditions, la fonction de corrélation $\rho(d)$ est décroissante et telle que :

$$
\rho(d) \# 0 \text { si } d>d \max
$$




\section{4. - Calcul de la précision d'un sondage systématique}

2.41. Si la forêt inventoriée est un ensemble de $\mathrm{N}$ placettes, et si l'échantillon systématique observé forme un réseau rectangulaire comportant $n$ placettes avec $\frac{\mathrm{N}}{n}=k$ entier, $f=\frac{1}{k}$ est le taux de sondage. Il y a alors $k$ échantillons systématiques possibles ayant la même configuration géométrique, aux légères variations près dues à la forme de la frontière de la forêt.

Si $x_{\mathrm{ij}}{ }^{(1)}$ est le nombre d'arbres d'une essence donnée existant effectivement sur la placette $(i, j)$ et si $\overline{\bar{x}}=\frac{1}{\mathrm{~N}} \sum_{\mathrm{ij}} x_{\mathrm{ij}}$ (la sommation étant faite par rapport à toutes les placettes de la forêt) on estime $\overline{\bar{x}}$ par $\bar{x}=\frac{1}{n} \sum_{\mathrm{ij}} x_{\mathrm{ij}}$ qui est le nombre moyen d'arbres de la même essence par placette de l'échantillon observé (la sommation se faisant maintenant par rapport aux placettes de l'échantillon).

Sans hypothèses supplémentaires, il est impossible de se faire une idée de l'erreur commise en remplaçant $\overline{\bar{x}}$ par $\bar{x}$.

Mais en introduisant l'hypothèse du processus stationnaire isotrope du second ordre, on peut considérer comme un indicateur de la précision du résultat obtenu :

$$
\sigma_{\mathrm{sy}}^{2}=\mathrm{E}\left[\frac{1}{k} \sum_{p=1}^{p=\mathrm{k}}\left(\overline{\mathrm{X}}_{\mathrm{p}}-\overline{\overline{\mathrm{X}}}\right)^{2}\right]
$$

où $\mathrm{E}$ indique l'espérance par rapport à la loi du processus :

et $\bar{X}_{p}$ et $\overline{\bar{X}}$ les variables aléatoires suivantes :

$\overline{\mathrm{X}}_{\mathrm{p}}=\frac{1}{\mathrm{n}} \sum_{\mathrm{ij}} \mathrm{X}_{\mathrm{ij}}$ (la sommation se faisant par rapport aux placettes de l'échantillon systématique $p, 1 \leqslant p \leqslant k$ ).

$\overline{\bar{X}}=\frac{1}{N} \sum_{\mathrm{ij}} \mathrm{X}_{\mathrm{ij}}$ (la sommation se faisant par rapport aux placettes de la forêt entière).

Ce schéma peut se résumer ainsi :

$\left(\bar{X}_{p}-\overline{\bar{X}}\right)^{2}$ a pour espérance au sens du processus $E\left(\bar{X}_{p}-\overline{\bar{X}}\right)^{2}$

qui a pour espérance au sens du sondage :

$$
\sigma_{\text {sy }}^{2}=\frac{1}{\mathrm{k}} \sum_{p=1}^{p=\mathrm{k}} \mathrm{E}\left(\overline{\mathrm{X}}_{\mathrm{p}}-\overline{\overline{\mathrm{X}}}\right)^{2}
$$

(1) $x_{i j}$ est la réalisation de la variable aléatoire $X_{i j}$ définie au $\S 23$. 
2.42. Calcul de $\sigma_{\mathrm{sy}}^{2}$ en fonction de $\rho$, fonction de corrélation du processus, et de la variance du processus.

Posons :

$\mathrm{S}_{p}^{2}=\frac{1}{n} \sum_{i j}\left(\mathrm{X}_{i j}-\overline{\mathrm{X}}_{p}\right)^{2}$ (la sommation se faisant par rapport aux placettes de l'échantillon systématique $p$ ),

$\Sigma^{2}=\frac{1}{\mathrm{~N}} \sum_{\mathrm{ij}}\left(\mathrm{X}_{\mathrm{ij}}-\overline{\overline{\mathrm{X}}}\right)^{2}$ (la sommation se faisant par rapport aux placettes de la forêt entière).

La décomposition de $\Sigma^{2}$ correspondant à la partition de la forêt en $k$ échantillons systématiques s'écrit :

$$
\Sigma^{2}=\frac{1}{k} \sum_{p=1}^{p=k} \mathrm{~S}_{p}^{2}+\frac{1}{k} \sum_{p=1}^{p=\mathrm{k}}(\overline{\mathrm{X}} p-\overline{\overline{\mathrm{X}}})^{2}
$$

On démontre que :

$$
\mathrm{E}\left(\Sigma^{2}\right)=\frac{\mathrm{N}-1}{\mathrm{~N}} \sigma^{2}(1-\overline{\bar{\rho}})
$$

en désignant par $\underset{\rho}{=}=\frac{1}{\mathrm{~N}(\mathrm{~N}-1)} \sum_{i, i=u, v} \rho\left(\sqrt{(i-u)^{2}+(j-v)^{2}}\right)$

c'est-à-dire la corrélation moyenne entre deux placettes distinctes de la forêt.

$\sqrt{(i-u)^{2}+(j-v)^{2}}$ étant la distance géométrique entre les placettes $(i, j)$ et $(u, v)$.

On démontre de même que :

$\mathrm{E}\left\lceil\frac{1}{n} \sum_{i, j}\left(\mathrm{X}_{i j}-\overline{\mathrm{X}} p\right)^{2}\right\rceil$, la sommation se faisant par rapport aux placettes de l'échantillon systématique $p$, peut s'écrire :

$$
\mathrm{E}\left|\frac{1}{n} \sum_{i j}\left(\mathrm{X}_{i j}-\mathrm{X}_{p}\right)^{2}\right|=\mathrm{E}\left(\mathrm{S}_{p}^{2}\right)=\frac{n-1}{n} \sigma^{2}\left(1-\bar{\rho}_{p}\right)
$$

$\bar{\rho}_{p}$ étant la corrélation moyenne entre deux placettes distinctes de l'échantillon systématique $\mathrm{p}$.

Mais la fonction de corrélation $\rho$ ne dépend que de la distance et les échantillons systématiques se déduisent par translation. Donc $\bar{\rho}_{p}$ ne dépend pas de $p$ et peut être noté $\bar{\rho}$.

D'où :

$$
\mathrm{E}\left\lceil\frac{1}{k} \sum_{\mathrm{p}=1}^{\mathrm{p}=\mathrm{k}} \mathrm{S}_{p}^{2}\right\rceil=\frac{1}{k} \sum_{\mathrm{p}=1}^{\mathrm{p}=\mathrm{k}} \mathrm{E}\left(\mathrm{S}_{p}^{2}\right)=\frac{1}{k} k \sigma^{2} \frac{n-1}{n}(1-\bar{\rho})=\frac{n-1}{n} \sigma^{2}(1-\bar{\rho})
$$

Et en prenant l'espérance par rapport au processus des deux membres de l'égalité (1), on obtient à partir de (2) et (4) : 


$$
\sigma_{s y}^{2}=\sigma^{2}\left[\frac{\mathrm{N}-1}{\mathrm{~N}}(1-\overline{\bar{\rho}})-\frac{n-1}{n}(1-\bar{\rho})\right]
$$

Cette formule peut s'écrire :

$$
\frac{\sigma_{s y}^{2}}{\sigma^{2}}=\frac{1-f}{n}+\bar{\rho}\left(1-\frac{1}{n}\right)-\bar{\rho}\left(1-\frac{1}{\mathrm{~N}}\right)
$$

où $\frac{1-f}{n}$ est le coefficient de $\sigma^{2}$ dans la formule du sondage aléatoire.

On voit donc que s'introduit un terme supplémentaire dont le signe, si $n$ est suffisamment grand, dépend de la différence entre $\overline{\bar{\rho}}$ et $\bar{\rho}$

\subsection{Estimation de $\sigma_{s y}^{2} \grave{a}$ partir de l'échantillon systématique.}

Pour estimer $\sigma_{s y}^{2}$ il faut estimer $\sigma^{2}$, $\overline{\bar{\rho}}$ et $\bar{\rho}$ à partir de l'échantillon systématique.

$\mathrm{Si}: \mathrm{S}_{p}^{2}=\frac{1}{n} \sum_{i j}^{\top}\left(\mathrm{X}_{i j}-\overline{\mathrm{X}} p\right)^{2}$ (la sommation se faisant par rapport aux placettes de l'échantillon systématique), on a, d'après (4) :

$$
\mathrm{E}\left(\mathbf{S}_{p}^{2}\right)=\frac{n-1}{n} \sigma^{2}(1-\bar{\rho}) .
$$

Donc : $s^{2}=\frac{1}{n} \sum_{i j}\left(x_{i j}-\bar{x}\right)^{2}$, où $x_{i j}$ est la réalisation de $\mathrm{X}_{i j}$ sur la placette $(i, j)$ de l'échantillon observé et $\bar{x}$ la moyenne arithmétique des $x_{i j}$, permet d'estimer sans biais $\sigma^{2}$ par : $\frac{n}{n-1} s^{2} \frac{1}{1-\bar{\rho}}$

Si on ne connaît pas $\rho(d)$, on peut en avoir une estimation $r(d)$, calculée par des méthodes d'ajustement appliquées au corrélogramme obtenu en observant tous les couples de placettes possibles de l'échantillon. La fonction $r(d)$ permet de calculer $\bar{r}$ et $\overline{\bar{r}}$ estimations de $\bar{\rho}$ et $\overline{\bar{\rho}}$.

On peut donc estimer $\sigma_{s y}^{2}$ par :

$$
\sigma_{s y}^{2}=s^{2}\left\lceil\frac{\mathrm{N}-1}{\mathrm{~N}-k} \frac{1-\overline{\bar{r}}}{1-\bar{r}}-1\right\rceil
$$

De cette relation on déduit que, si $\bar{r}=\overline{\bar{r}}=0$, ce qui a lieu en particulier dans le cas d'une distribution « au hasard » du nombre de tiges, on a :

$$
\hat{\sigma}_{s y}^{2}=s^{2}\left(\frac{\mathrm{N}-1}{\mathrm{~N}-k}-1\right)=s^{2} \frac{k-1}{\mathrm{~N}-k}=s^{2} \frac{1-f}{n-1}
$$

ce qui est la formule élémentaire du sondage aléatoire, ( $f$ désignant le taux de sondage $\left.\frac{1}{k}\right)$. 
On retrouve aussi cette formule (7) si $\bar{r}=\overline{\bar{r}}$ c'est-à-dire si l'échantillon et la population ont la même corrélation moyenne.

Par contre, cette formule (7) conduit à une surestimation de la variance lorsque $\bar{r}<\overline{\bar{r}}$, c'est-à-dire lorsque l'échantillon a une corrélation moyenne inférieure à celle de la forêt.

\section{III. - MÉTHODES DE CALCUL}

\section{1. - Détermination d'une fonction de corrélation $\rho=p(d)$}

\subsection{Cas d'un inventaire complet.}

Si on a pu effectuer un inventaire complet d'une forêt, on peut alors estimer avec une précision acceptable les valeurs du coefficient de corrélation pour toutes les valeurs de la distance et construire ainsi un corrélogramme.

On peut par exemple procéder ainsi : on fixe une direction donnée et on prend l'ensemble des couples de parcelles de la population situées à la distance $d_{i}$ l'une de l'autre dans cette direction; si $x$ désigne la valeur du paramètre dans la première parcelle et $y$ cette valeur dans la deuxième, $p\left(d_{i}\right)$ est estimé par le coefficient de corrélation calculé à partir de tous les couples $x, y$ ainsi obtenus.

Si alors on peut admettre qu'une forêt sondée présente à peu près la même distribution du paramètre étudié que celle inventoriée complètement, on dispose ainsi d'un corrélogramme empirique qu'on pourra utiliser pour le calcul des corrélations moyennes $\overline{\bar{r}}$ et $\bar{r}$ dans la forêt sondée et dans l'échantillon.

\subsection{Estimation des paramètres de corrélogramme.}

$$
\rho(d)=p e^{-a d}+q e^{-b d}, a>o, b>o .
$$

Nous avons déjà signalé que $\rho(d)=e^{-a d}(a>o)$ était une fonction de corrélation d'un processus isotrope plan; on remarquera que :

$$
\rho(0)=p+q=1
$$

\subsection{Méthode empirique du papier semi-logarithmique.}

On porte sur papier semi-logarithmique les points $\left[d_{i}, \rho\left(d_{i}\right)\right]$. Si par exemple $b$ est notablement supérieur à $a$, on aura :

$$
\rho(d) \approx p e^{- \text {ad }} \text { pour } d \text { assez grande. }
$$

Dans ce cas les points obtenus sont alignés et par simple lecture on détermine $a$ et $p$.

Sur le même papier, on mesure et on reporte la différence $\left[\rho(d)-p e^{- \text {ad }}\right]$ et les points obtenus peuvent être à nouveau alignés sur une droite, ce qui permet de déterminer $q$ et $b$. 
3.122. Méthode des totaux partiels.

$$
\text { On pose : } \begin{aligned}
v & =e^{-\mathrm{a}} \\
w & =e^{-\mathrm{b}}
\end{aligned}
$$

Prenant l'échantillon de points $\left(d_{i}, \rho_{i}\right)$ de taille $4 n$ ( $i$ variant de 0 à $4 n-1$ ), on écrit les relations simples suivantes :

$$
\begin{aligned}
& \mathrm{S}_{1}=\sum_{i=0}^{n-1}\left[\rho\left(d_{i}\right)\right]=p \quad \frac{\left(1-v^{\mathrm{n}}\right)}{1-v}+q \frac{1-w^{\mathrm{n}}}{1-w}, \\
& \mathrm{~S}_{2}=\sum_{i=n}^{2 n-1}\left[\rho\left(d_{i}\right)\right]=p v^{\mathrm{n}} \frac{1-v^{\mathrm{n}}}{1-v}+q w^{\mathrm{n}} \frac{1-w^{\mathrm{n}}}{1-w}, \\
& \mathrm{~S}_{3}=\sum_{i=2 n}^{3 n-1}\left[\rho\left(d_{i}\right)\right]=p v^{2 \mathrm{n}} \frac{1-v^{\mathrm{n}}}{1-v}+q w^{2 \mathrm{n}} \frac{1-w^{\mathrm{n}}}{1-w}, \\
& \mathrm{~S}_{4}=\sum_{i=3 n}^{4 n-1}\left[\rho\left(d_{i}\right)\right]=p v^{3 \mathrm{n}} \frac{1-v^{\mathrm{n}}}{1-v}+q w^{3 \mathrm{n}} \frac{1-w^{\mathrm{n}}}{1-w} .
\end{aligned}
$$

Si l'on pose : $\mathrm{A}_{0}=1$

$\mathbf{A}_{1}=w^{n}+v^{\mathrm{n}}$
$\mathbf{A}_{2}=w^{\mathrm{n}} v^{\mathrm{n}}$

on a les relations :

$$
\begin{aligned}
& A_{2} S_{1}-A_{1} S_{2}+A_{0} S_{3}=0 \\
& A_{2} S_{2}-A_{1} S_{3}+A_{0} S_{4}=0
\end{aligned}
$$

qui permettent de déterminer $A_{1}$ et $A_{2}$.

L'équation du second degré :

$$
x^{2}-x \mathrm{~A}_{1}+\mathrm{A}_{2}=0
$$

permet de déterminer $w$ et $v$, donc $a$ et $b$.

On détermine $p$ et $q$ grâce aux relations donnant $\mathbf{S}_{1}$ et $\mathbf{S}_{2}$.

3.123. Méthode des intégrations numériques.

Cette méthode est exposée par Stéphen O. Foss dans le numéro de décembre 1970 de la revue Biometrics.

\section{2. - Calcul des corrélations moyennes}

On calcule la somme des corrélations et on en déduit immédiatement la corrélation moyenne. 
3.21. Cas d'une forêt de forme rectangulaire de côtés $\mathrm{L}_{1}$ et $\mathrm{L}_{2}$.

\subsection{Calcul de $\bar{r}$.}

Nous calculerons les sommes de corrélations $\Sigma_{1}, \Sigma_{2}, \Sigma_{3}$ :

$\Sigma_{1}$ : somme des corrélations entre parcelles séparées par des distances entières;

$\Sigma_{2}$ : somme des corrélations entre parcelles séparées par des distances de la forme $n \sqrt{2}$, $n$ entier;

$\Sigma_{3}$ : somme des corrélations entre parcelles séparées par des distances de la forme $\sqrt{n^{2}+m^{2}}, n$ et $m$ entiers.

La somme des corrélations n'est autre que :

$$
\Sigma_{1}+\Sigma_{2}+\Sigma_{3}
$$

a) Calcul de $\Sigma_{1}$

Si $d$ est un entier $i$, il y a :

$$
\mathrm{L}_{1}\left(\mathrm{~L}_{2}-i\right)+\mathrm{L}_{2}\left(\mathrm{~L}_{1}-i\right)
$$

couples de parcelles séparées par la distance $d$.

D'où :

$$
\Sigma_{2}=\sum_{i}\left[\mathrm{~L}_{1}\left(\mathrm{~L}_{2}-i\right)+\mathrm{L}_{2}\left(\mathrm{~L}_{1}-i\right)\right] r(i) \quad\left(1 \leqslant i \leqslant d_{\max }\right)
$$

b) Calcul de $\Sigma_{2}$

De même si $d$ est de la forme $i \sqrt{2}$ il y a :

$$
2\left(\mathrm{~L}_{1}-i\right)\left(\mathrm{L}_{2}-i\right) \text { couples correspondants. }
$$

D'où :

$$
\Sigma_{2}=\sum_{i} 2\left(\mathrm{~L}_{1}-i\right)\left(\mathrm{L}_{2}-i\right) r(i \sqrt{2}) \quad\left(1 \leqslant i \leqslant \frac{d_{\max }}{\sqrt{2}}\right)
$$

c) Calcul de $\Sigma_{3}$

De même si $d$ est de la forme $d=\sqrt{i^{2}+j^{2}}(i \neq j)$ il y a :

$$
2\left(\mathrm{~L}_{1}-i\right)\left(\mathrm{L}_{2}-j\right)+2\left(\mathrm{~L}_{1}-j\right)\left(\mathrm{L}_{2}-i\right) \text { couples correspondants. }
$$

D'où :

$$
\begin{gathered}
\Sigma_{3}=\sum_{i, j} 2\left[\left(\mathrm{~L}_{1}-i\right)\left(\mathrm{L}_{2}-j\right)+\left(\mathrm{L}_{1}-j\right)\left(\mathrm{L}_{2}-i\right)\right] r\left(\sqrt{i^{2}+j^{2}}\right), \\
\left(1 \leqslant i \leqslant d_{\text {max }} \text { et } 1+i \leqslant j \leqslant \mathscr{E}\left(\sqrt{d^{2} \text { max }-i^{2}}\right) \text { avec } \mathscr{E}(x)=\text { partie entière de } x .\right)
\end{gathered}
$$

\subsection{Calcul de $\bar{r}$.}

Soit un échantillon systématique non en quinconce obtenu en prélevant une parcelle sur $u$ dans le sens de la longueur et une parcelle sur $v$ dans le sens de la largeur ( $u$ et $v$ entiers). 
Les calculs précédents se transposent à l'échantillon en remplaçant $\mathrm{L}_{1}$ et $\mathrm{L}_{2}$ respectivement par :

$$
\begin{aligned}
& \frac{\mathrm{L}_{1}}{u} \text { et } \frac{\mathrm{L}_{2}}{v} \text { et } i, j \text { et } \sqrt{i^{2}+j^{2}} \text { respectivement par } \\
& i u, j v \text { et } \sqrt{i_{2} u^{2}+j^{2} v^{2}} \text {. }
\end{aligned}
$$

Si l'échantillon systématique est en quinconce, on appliquera l'algorithme exposé ci-après.

\subsection{Cas d'une forêt de forme quelconque.}

Le calcul des corrélations moyennes $\bar{r}$ et $\bar{r}$ se complique considérablement dans le cas d'une forêt de forme quelconque. L'algorithme ci-dessous n'est utilisable qu'avec l'aide d'un ordinateur. A titre d'indication, un essai fait sur une forêt inscrite dans un carré de 6400 parcelles a demandé 10 secondes de calcul sur IBM 360-91.

- On commence par inclure la forêt dans un rectangle dont les côtés sont parallèles à ceux des parcelles, ceci pour faciliter la mise en mémoire de la forme de la forêt; on rentre en mémoire le rectangle découpé en parcelles (une parcelle étant notée 1 si elle appartient à la forêt, 0 sinon).

- Ayant choisi un sens de parcours des parcelles du rectangle, on calcule la somme des corrélations entre une parcelle de la forêt et toutes les suivantes situées dans la forêt (aucun calcul n'est fait si 1'ordinateur trouve un 0 dans la parcelle).

Le calcul est simplifié par le fait que $r(d)$ est négligeable si $d>d_{\text {max }}$. Par suite, chaque fois que dans le calcul $d>d_{\max }$, l'ordinateur change soit de ligne, soit de colonne.

$$
\begin{aligned}
& \text { Exemple : } d_{\max }=3 \\
& \begin{array}{llllllllllll}
0 & 0 & 0 & 0 & * & * & * & * & 0 & 0 \\
0 & 0 & * & * & * & * & * & * & 0 & 0 \\
0 & 0 & * & * & * & * & * & * & 0 & 0 \\
0 & 0 & * & * & * & * & * & * & 0 & 0 \\
0 & 0 & 0 & 0 & 0 & 0 & 0 & 0 & 0 & 0
\end{array}
\end{aligned}
$$

Ainsi, quand la parcelle $[*$ est fixée, la somme des corrélations avec cette parcelle ne s'effectue qu'en prenant les parcelles en astérisque. Certaines de ces parcelles $(\stackrel{*}{=})$ n'interviennent pas dans le calcul, car elles sont situées à une distance supérieure à $d_{\text {max }}$.

\section{IV. - APPLICATION A UN INVENTAIRE DE FORÊT DENSE TROPICALE}

\subsection{Expérimentation réalisée}

Comme on l'a remarqué au début de cet article, les avantages des dispositifs systéma- 
tiques sont particulièrement appréciables dans les inventaires de forêt dense tropicale, milieu végétal où les problèmes de repérage et d'accessibilité imposent pratiquement toujours l'adoption de tels dispositifs.

Une application expérimentale de la méthode proposée a été rendue possible par l'existence d'un ensemble de données provenant d'un inventaire exhaustif réalisé dans un peuplement de forêt dense semi-décidue au Cameroun Oriental (région de Bertoua).

Une surface pratiquement plane de forêt dense rectangulaire de 1440 hectares, de $6 \mathrm{~km}$ de long sur 2,4 km de large a été découpée par un quadrillage de layons et de " traces » (layons sommaires) en 5760 parcelles carrées contigües de 25 ares (50 mètres sur 50 mètres). Les tiges des dix essences commerciales les plus importantes de cette région ont été recensées parcelle par parcelle, par classes de surface terrière à partir de $36 \mathrm{~cm}$ de diamètre. Les paramètres retenus pour l'application de cette étude sont les nombres de tiges de diamètre supérieur à $36 \mathrm{~cm}$ par parcelle dont les caractéristiques sont les suivantes :

\begin{tabular}{|c|c|c|c|c|}
\hline $\begin{array}{c}\text { Nom } \\
\text { commercial }\end{array}$ & $\begin{array}{c}\text { Essence } \\
\text { Nom scientifique }\end{array}$ & Famille & $\begin{array}{c}\text { Moyenne } \\
\text { vraie }\end{array}$ & $\begin{array}{l}\text { Coefficient } \\
\text { de variation } \\
(\%)\end{array}$ \\
\hline Ayous (SAMBA) & $\begin{array}{l}\text { Triplochiton } \\
\text { scleroxylon }\end{array}$ & Sterculiacée & 0,202 & 330 \\
\hline Limba & $\begin{array}{c}\text { Terminalia } \\
\text { superba }\end{array}$ & Combrétacée & 0,547 & 184 \\
\hline Sapelli & $\begin{array}{l}\text { Entandrophragma } \\
\text { cylindricum }\end{array}$ & Méliacée & 0,212 & 232 \\
\hline \multicolumn{3}{|c|}{$\begin{array}{l}\text { Ensemble de } 10 \text { essences commerciales comprenant les trois précé } \\
\text { dentes. }\end{array}$} & 1,328 & 111 \\
\hline
\end{tabular}

L'Ayous a une distribution contagieuse par « bouquets », le Limba également, mais de façon moins marquée et plus diffuse. Le Sapelli est celle des essences étudiées dont la répartition du nombre de tiges par parcelle est la plus proche d'une distribution «au hasard » (distribution de Poisson) mais sa densité est faible. Enfin le groupe des 10 essences a une distribution plus « régulière » provenant en particulier de ce que des compensations s'établissent entre les densités des essences qui composent ce groupe.

En particulier, on peut noter que 1'Ayous et le Limba, lorsqu'ils sont abondants, ont tendance à exclure les huit autres essences.

Disposant ainsi d'un ensemble complet de données pour la surface étudiée, on a pu l'utiliser pour expérimenter la méthode proposée ci-dessus. Plus précisément on a procédé avec ces données aux études suivantes (relativement aux quatre paramètres indiqués plus haut) :

- détermination d'une fonction de corrélation,

— étude de la validité de l'estimation de la précision par la méthode proposée,

— étude de l'efficacité relative des différents dispositifs systématiques. 


\section{2. - Détermination d'une fonction de corrélation}

4.21. Détermination des valeurs du coefficient de corrélation pour les différentes valeurs entières et irrationnelles de la distance d dans le cas d'un comptage complet.

Les valeurs entières de $d$ correspondent à des translations parallèlement aux deux côtés du rectangle de quadrillage, les valeurs irrationnelles correspondant à des déplacements obliques.

Dans un premier temps, on a pris l'ensemble des couples de parcelles situées à une distance $d$ entière comprise entre 1 et 30 (en unités égales au côté des parcelles) et on a déterminé la valeur du coefficient de corrélation pour chaque distance $d$ à partir de tous les couples. Les valeurs trouvées sont indiquées dans le tableau ci-dessous.

TABLEAU 1

Valeurs de $\mathrm{r}$ pour des distances entières entre 1 et 30

\begin{tabular}{|c|c|c|c|c|c|}
\hline $\begin{array}{l}\text { Distance } \\
\quad d\end{array}$ & $\begin{array}{c}\text { Nombre } \\
\text { de couples }\end{array}$ & Ayous & Limba & SAPELLI & 10 EsSENCES \\
\hline 1 & 11.352 & 0,451 & 0,371 & 0,108 & 0,294 \\
\hline 2 & $11 \cdot 184$ & 0,266 & 0,277 & 0,061 & 0,190 \\
\hline 3 & 11.016 & 0,184 & 0,224 & 0,032 & 0,129 \\
\hline 4 & 10.848 & 0,160 & 0,199 & 0,044 & 0,113 \\
\hline 5 & 10.680 & 0,134 & 0,163 & 0,023 & 0,072 \\
\hline 6 & 10.512 & 0,101 & 0,142 & 0,013 & 0,055 \\
\hline 7 & 10.344 & 0,083 & 0,121 & 0,003 & 0,040 \\
\hline 8 & 10.176 & 0,065 & 0,120 & 0,006 & 0,038 \\
\hline 9 & 10.008 & 0,064 & 0,100 & 0,003 & 0,030 \\
\hline 10 & 9.840 & 0,056 & 0,093 & 0,000 & 0,028 \\
\hline 12 & 9.504 & 0,039 & 0,068 & très faiblement & 0,015 \\
\hline 15 & 9.000 & $-0,005$ & 0,056 & positif ou négatif & $-0,013$ \\
\hline 17 & 8.664 & & 0,049 & & très faiblement \\
\hline 21 & 7.992 & $\begin{array}{l}\text { très faiblement } \\
\text { positif ou négatif }\end{array}$ & 0,031 & & positif ou négatif \\
\hline $\begin{array}{l}24 \\
27 \\
30\end{array}$ & $\begin{array}{l}7.488 \\
6.984 \\
6.480\end{array}$ & $(\mathrm{r}<0,02)$ & $\begin{array}{r}0,016 \\
-0,004 \\
-0,006\end{array}$ & & $(\mathrm{r}<0,05)$ \\
\hline
\end{tabular}

La figure 1 ci-après reproduit graphiquement les résultats du tableau précédent. Elle fait bien apparaître que :

- le coefficient de corrélation décroît avec la distance $d$,

- pour une essence donnée il existe une distance au-delà de laquelle le coefficient de corrélation est nul ou négligeable en valeur absolue.

Pour toutes les valeurs irrationnelles, plutôt que d'effectuer un lissage, on a préféré appliquer un programme d'interpolation linéaire entre les entiers successifs. Ce sont les valeurs de $\rho$ ainsi obtenues qui ont été utilisées dans le calcul de $\hat{\sigma}_{s y}^{2}$ pour le peuplement étudié. Le seul inconvénient de l'interpolation linéaire est la valeur assez approximative qu'on obtient pour $d=\sqrt{2}$ du fait de la décroissance rapide de $p$ entre les valeurs 1 et 2 . Aussi est-il préférable de calculer directement $\rho$ pour $d=\sqrt{ } 2$ en prenant tous les couples correspondant à cette distance. 
$r$ Coefficient de corrélation

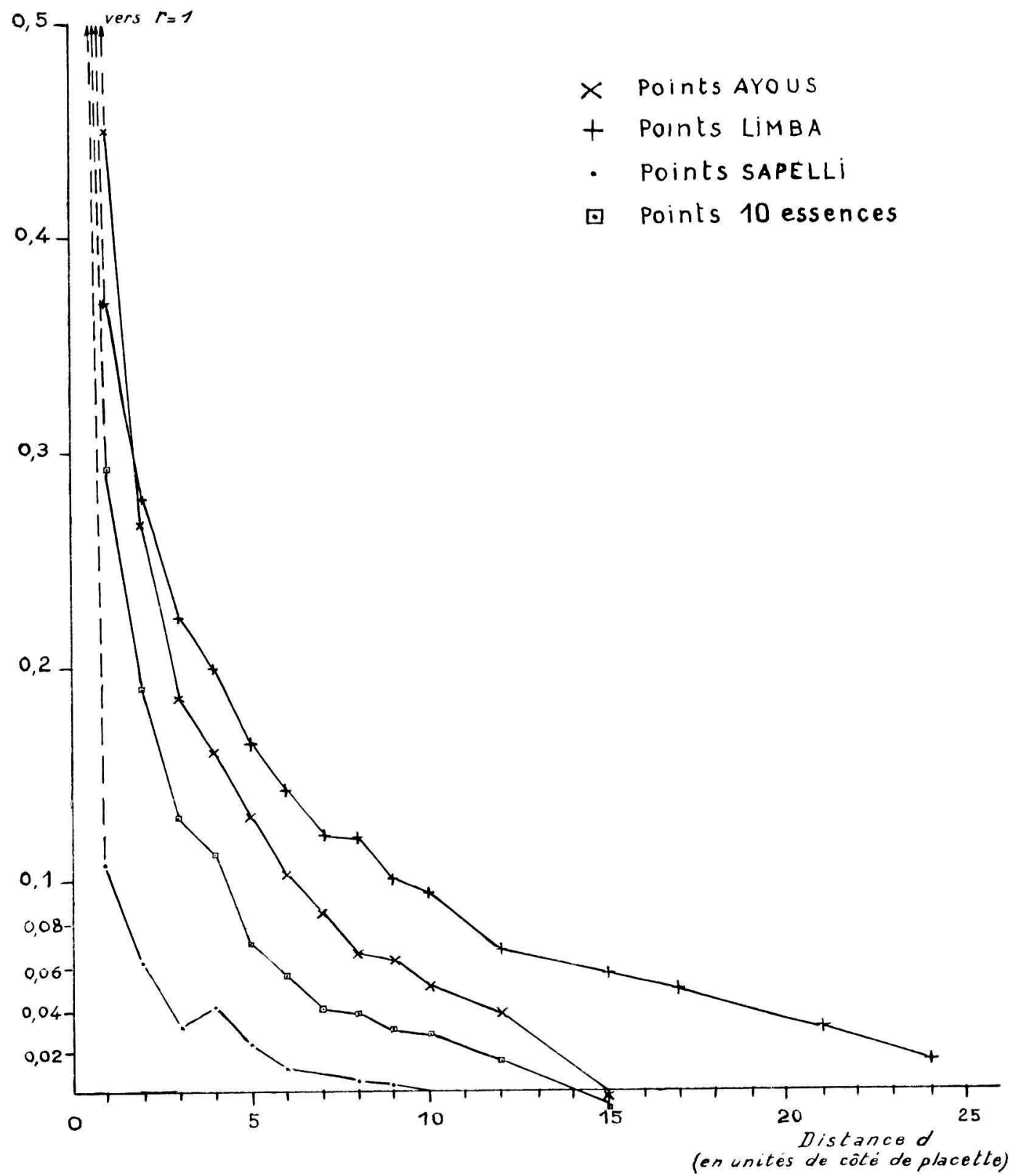

Fig. 1. - Variation du coefficient de corrélation en fonction de la distance $d$ pour les quatre paramètres étudiés

FIG. 1. - Correlogrammes for the four parameters 
Les valeurs trouvées pour $\overline{\bar{r}}$ (coefficient de corrélation moyen sur l'ensemble de la popu lation) sont les suivantes :

$$
\begin{array}{ll}
\text { Ayous } & : \overline{\bar{r}}=0,0067 \\
\text { Limba } & : \overline{\bar{r}}=0,0158 \\
\text { Sapelli } & : \overline{\bar{r}}=0,0008 \\
\text { 10 essences } & : \overline{\bar{r}}=0,0040
\end{array}
$$

Le tableau 2 donne quelques valeurs de $\bar{r}$ (coefficient de corrélation moyen sur l'échantillon) pour différents types d'échantillons systématiques caractérisés par les quantités $u$ et $v$ définies comme suit :

- $u$ est l'entier inverse du rapport du côté d'une parcelle à la distance séparant celle-ci de la parcelle de l'échantillon immédiatement voisine dans le sens de la plus grande longueur de la forêt,

- $v$ est 1 'entier inverse du rapport semblable du côté de la parcelle à la distance séparant la parcelle à celle immédiatement voisine dans la direction perpendiculaire à la précédente (en supposant que le dispositif systématique n'est pas « en quinconce »),

\begin{tabular}{|c|c|c|c|c|c|c|c|}
\hline$u$ & $v$ & $\stackrel{u v}{=} k$ & $\begin{array}{l}f(\%) \\
=\frac{100}{k}\end{array}$ & $\begin{array}{c}\underset{\bar{r}}{\text { Ayous }} \\
(\overline{\bar{r}}=0,0067)\end{array}$ & $\begin{array}{c}\underset{\bar{r}}{\operatorname{LimBA}} \\
(\overline{\bar{r}}=0,0158)\end{array}$ & $\begin{array}{c}\operatorname{SAPELLI}_{\bar{r}} \\
(\overline{\bar{r}}=0,0008)\end{array}$ & $\begin{array}{l}10 \text { ESSENCES } \\
\bar{r} \\
(\overline{\bar{r}}=0,0040)\end{array}$ \\
\hline 1 & & & & & & & 0098 \\
\hline \multirow[t]{2}{*}{2} & $\begin{array}{l}24 \\
48\end{array}$ & $\begin{array}{l}24 \\
96\end{array}$ & $\begin{array}{l}4,17 \\
1,04\end{array}$ & $\begin{array}{l}0,0136 \\
0,0235\end{array}$ & $\begin{array}{l}0,0218 \\
0,0358\end{array}$ & $\begin{array}{l}0,0024 \\
0,0037\end{array}$ & 0,0144 \\
\hline & 16 & 32 & 3,12 & 0,0077 & 0,0230 & 0,0011 & 0,0047 \\
\hline \multirow[t]{2}{*}{3} & 48 & 144 & 0,69 & 0,0197 & 0,0324 & 0,0026 & 0,0118 \\
\hline & 16 & 48 & 2,08 & 0,0065 & 0,0159 & 0,0008 & 0,0039 \\
\hline \multirow[t]{2}{*}{4} & 16 & 64 & 1,56 & 0,0056 & 0,0155 & 0,0006 & 0,0033 \\
\hline & 8 & 32 & 3,12 & 0,0052 & 0,0149 & 0,0005 & 0,0029 \\
\hline \multirow[t]{3}{*}{5} & 16 & 80 & 1,25 & 0,0047 & 0,0150 & 0,0005 & 0,0027 \\
\hline & 8 & 40 & 2,50 & 0,0054 & 0,0145 & 0,0004 & 0,0028 \\
\hline & 6 & 30 & 3,33 & 0,0053 & 0,0148 & 0,0004 & 0,0030 \\
\hline \multirow[t]{3}{*}{6} & 16 & 96 & 1,04 & 0,0044 & 0,0141 & 0,0004 & 0,0025 \\
\hline & 8 & 48 & 2,08 & 0,0044 & 0,0143 & 0,0003 & 0,0025 \\
\hline & 6 & 36 & 2,78 & 0,0055 & 0,0144 & 0,0004 & 0,0031 \\
\hline
\end{tabular}

avec $u v=k=\frac{1}{f}$ inverse du taux de sondage.

TABLEAU 2

Valeur de $\bar{r}$ pour quelques échantillons systématiques

On peut remarquer d'une manière générale que $\bar{r}$ décroît lorsque $u$ augmente, et qu'il varie de la même manière pour les quatre paramètres.

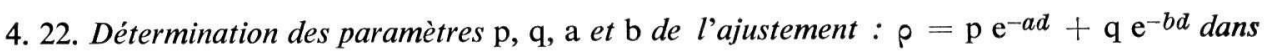
le cas général d'un inventaire par sondage.

On a appliqué la méthode graphique en coordonnées semi-logarithmiques décrite au paragraphe 3.121 à partir des valeurs de $\rho$ trouvées pour les entiers successifs. Bien que 
l'on se soit trouvé dans le cas d'un comptage complet, l'application de cette méthode a été profitable car elle en a montré l'intérêt pratique. On a pu déterminer sans ambiguïté les paramètres de l'ajustement pour trois des quatre caractéristiques numériques étudiées.

\section{3. - Validité de l'estimation de la précision par la méthode proposée}

On a déterminé pour chaque type d'échantillon systématique caractérisé par un couple donné de valeurs $u_{0}$, vo la valeur vraie de l'écart-type de la moyenne du sondage systématique, à savoir :

$$
\sigma_{s y_{0}}=\frac{1}{\sqrt{k}} \sqrt{\sum_{p=1}^{k}\left(x_{p}-\overline{\bar{x}}\right)^{2}} \quad\left(\operatorname{avec} k=u_{0} v_{0}\right)
$$

Pour chaque échantillon systématique correspondant à un type d'échantillon $\left(u_{0}\right.$, $\left.v_{0}\right)$ on a calculé :

- la moyenne $\overline{\mathrm{x}}_{p}$ de l'échantillon

- l'estimation $\hat{\sigma}_{s y}$ de l'écart-type de la moyenne de l'échantillon par la méthode proposée, à savoir (formule (6)) :

$$
\begin{aligned}
& \hat{\sigma}_{s y}=s \sqrt{\frac{\mathrm{N}-11-\bar{r}}{\mathrm{~N}-k}-1-\bar{r}}-1 \\
& \text { avec } s^{2}=\frac{1}{n_{i j}}\left(x_{i j}-\vec{x}_{p}\right)^{2}
\end{aligned}
$$

la sommation étant étendue aux unités de l'échantillon considéré,

- l'estimation $\mathrm{s}_{a}$ de l'écart-type de la moyenne de l'échantillon en supposant que l'échantillon est aléatoire, à savoir :

$$
s_{a}=s \sqrt{\frac{1-f}{n-1}}=s \sqrt{\frac{k-1}{\mathrm{~N}-k}}
$$

On a ensuite déterm iné pour chaque couple $u_{0}, v_{0}$ :

- le rapport $q=\frac{\hat{\sigma}_{s y}}{s_{a}}$ des estimations de l'écart-type de la moyenne par la méthode des processus et par celle consistant à admettre que l'échantillon est aléatoire,

- la proportion $\mathrm{P}_{a}$ des échantillons $\left(u_{0}, v_{0}\right)$ tels que la moyenne vraie $\overline{\bar{x}}$ est comprise dans l'intervalle $\left(\bar{x}_{p}-2 s_{a}, \bar{x}_{p}+2 s_{a}\right)$,

- la proportion $\mathbf{P}$ des échantillons tels que $\bar{x}$ est comprise dans l'intervalle $\left(x_{p}-2 \hat{\sigma}_{s y}\right.$, $\left.p+2 \hat{\sigma}_{s y}\right)$,

- la proportion $\mathrm{P}_{0}$ des échantillons tels que $\bar{x}$ est comprise dans l'intervalle $\left(\bar{x}_{p}-\right.$ $\left.2 \sigma_{s y_{0}}, \bar{x}_{p}+2 \sigma_{s y_{0}}\right)$.

Cette dernière proportion n'est pas strictement égale à $95 \%$, compte tenu que la norma- 


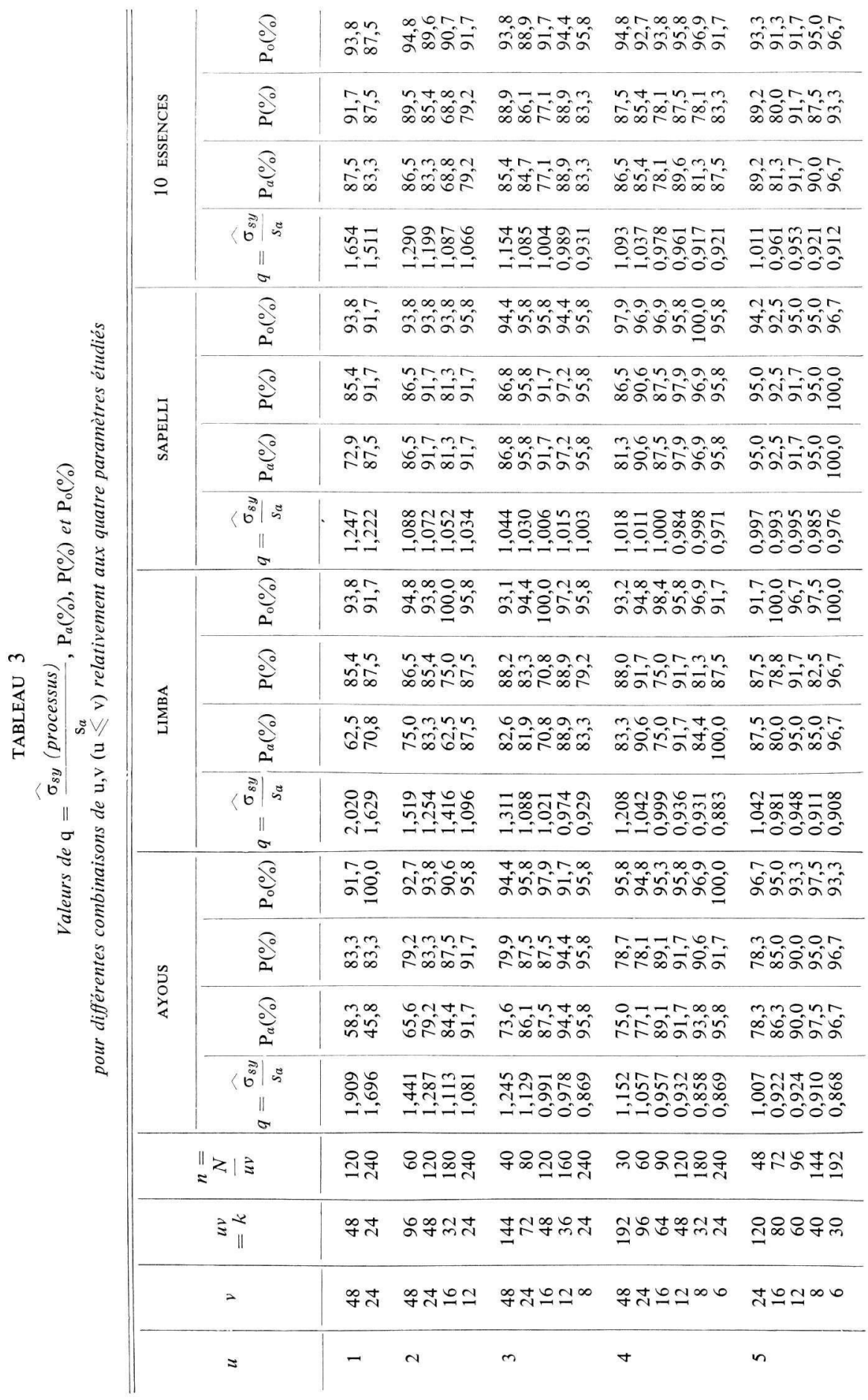


TABLEAU 3 (suite)

Valeurs de $\mathrm{q}=\frac{\widehat{\sigma}_{s y} \text { (processus) }}{\mathrm{s}_{\mathrm{o}}}, \mathrm{P} a(\%), \mathrm{P}(\%)$ et $\mathrm{P}(\%)$

pour différentes combinaisons de $\mathrm{u}, \mathrm{v}(\mathrm{u} \leqslant \mathrm{v})$ relativement aux quatre paramètres étudiés

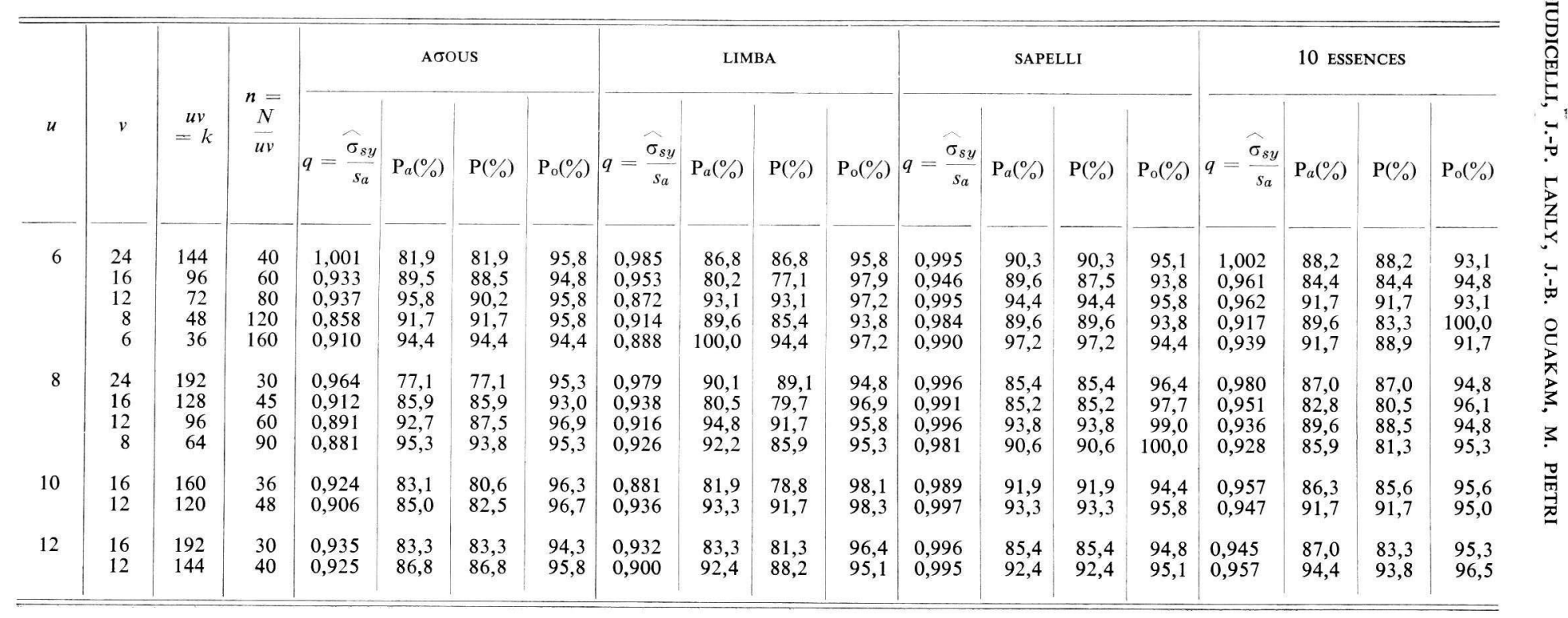


lité de la moyenne n'est pas exactement vérifiée du fait du faible effectif de certains échantillons et des faibles dimensions de la population.

La comparaison de $\mathrm{P}_{a}, \mathrm{P}$ et $\mathrm{P}_{\mathrm{o}}$ permet d'étudier la validité de la méthode proposée. Les valeurs de ces quantités sont données dans le tableau 3 pour les couples $(u, v)$ tels que $u \leqslant v$.

Le tableau 3 montre que l'estimation par la méthode de processus est :

— d'autant plus valable que le dispositif utilisé est plus «aplati» : cette méthode apporte une amélioration très sensible de l'estimation de la précision piur des faibles valeurs de $\mathbf{u}$ ( 1 à 4 ) et des fortes valeurs de v (24 et 48) ce qui, sur le plan pratique, est particulièrement intéressant en forêt tropicale, l'utilisation de dispositifs fortement aplatis étant préférée pour des raisons évidentes de coût;

- plus intéressante pour des paramètres fortement corrélés (Ayous et Limba) que pour les paramètres dont la distribution est plus proche d'une distribution « au hasard » (Sapelli notamment pour lequel les deux estimations donnent pratiquement toujours les mêmes résultats sauf pour $u=1$ et $v=48$ et 24).

Le tableau 3 montre le danger qu'il y a à estimer par la méthode habituelle la précision d'un échantillonnage systématique « aplati »: $\mathrm{P}_{a}$ est très inférieur à $\mathrm{P}_{0}$ pour $u$ très petit et $v$ très grand, particulièrement dans le cas de l'Ayous et du Limba.

Afin de rendre plus évident l'intérêt de cette méthode pour l'estimation de la précision dans le cas des dispositifs « aplatis » on a reporté, pour chaque paramètre (graphiques de la figure 2) les points

$$
\begin{aligned}
& \text { d'abscisse } x=\frac{\max (u, v)}{\min (u, v)} \text { (mesurant l'aplatissement du dispositif) } \\
& \text { et d'ordonnée } y=\frac{\mathrm{P}}{\mathrm{P}_{a}}
\end{aligned}
$$

Les nuages de points correspondant à l'Ayous et au Limba sont inclinés par rapport à l'axe des $x$, ce qui montre que plus le dispositif est aplati plus grand est $\mathrm{P}$ par rapport à $\mathrm{P}_{a}$, c'est-à-dire meilleure est l'estimation donnée $\left(\mathrm{P}_{a}<\mathrm{P}<\mathrm{P}_{\mathrm{o}}\right)$.

\section{4. — Étude de l'efficacité des différents dispositifs systématiques}

4.41. L'intérêt le plus grand de cette méthode dans son application aux inventaires de forêt dense tropicale est de permettre de calculer l'erreur de sondage dans le cas où la non-indépendance des unités entre elles, relativement aux paramètres étudiés — du fait de la distribution non « au hasard » de ceux-ci - interdit l'utilisation de la formule élémentaire du sondage strictement aléatoire. Or, pour des raisons évidentes de coût, les parcelles de l'échantillon sont peu espacées entre elles le long des layons qui les relient, afin de réduire la longueur d'ouverture de sous-bois par unité de surface sondée. Les calculs montrent qu'à taux de sondage donné ( $u v$ fixé), $\sigma_{s y}$, donc l'erreur de sondage, varie significativement lorsque $u$ et $v$ varient.

De même le coût augmente à taux de sondage égal lorsque $u$ augmente, c'est-à-dire 
lorsque 1'intervalle entre parcelles voisines sur une même ligne augmente. On est donc naturellement conduit, pour chaque paramètre étudié, à rechercher quel est le dispositif systématique optimal, c'est-à-dire celui qui, pour un coût imposé à l'avance, donne l'erreur de sondage minimum, ou qui, pour une marge d'incertitude fixée a priori, donne le coût minimum.

\subsection{Principe.}

a) On écrit que la marge d'incertitude relative au seuil 0,95 sur un paramètre donné $x$ est égale en pourcentage à :

$$
e(\%)=\frac{200}{\bar{x}} \sigma_{s y}
$$

A partir des résultats du tableau 44, et après analyse des valeurs calculées de $e(\%)$ pour les différents couples $u$ et $v$, on a déterminé par une régression progressive multiple des ajustements de la forme :

$u$ étant systématiquement plus petit que $v$.

$$
e(\%) \simeq \alpha u+\beta v+\gamma u v+\delta
$$

Pour chacun des paramètres étudiés, on a :

$$
\begin{aligned}
& e=\gamma_{1} u v+\delta_{1} \quad \text { avec } \mathrm{R}>0,80 \text { ( } \mathrm{R} \text { coefficient de corrélation multiple) } \\
& e=\beta_{2} v+\gamma_{2} u v+\delta_{2} \quad \text { avec } \mathrm{R}>0,98 \\
& e=\alpha u+\beta v+\gamma u v+\delta \quad \text { avec } \mathrm{R}>0,99
\end{aligned}
$$

b) On détermine une fonction de coût qui dans ce type d'inventaire peut s'écrire d'une manière simplifiée sous la forme :

$$
\mathrm{C}=\mathrm{K} \frac{u+a}{u v}(u<v)
$$

c) On fixe $e=e_{0}$ (ou $\mathrm{C}=\mathrm{C}_{0}$ ) et on déduit les valeurs de $u$ et $v$ qui minimisent $\mathrm{C}$ (ou $e$ ).

\subsection{Résultats.}

On trouve dans les deux hypothèses :

$$
u=u_{0}=\sqrt{\frac{a \beta}{\delta}}
$$

$v$ s'en déduisant à partir de $e=e_{0}=\alpha u_{0}+\beta v+\gamma u_{0} v+\delta$

(ou à partir de $\left.\mathrm{C}=\mathrm{C}_{0}=k \frac{u_{0}+a}{u v}\right)$

Avec des hypothèses admissibles sur la marge d'incertitude $e_{0}$ (ou sur le coût $\mathrm{C}_{0}$ ) on aboutit à des valeurs de $u$ comprises entre 1 et 4 . 


\section{V. - CONCLUSION}

Le modèle proposé fournit une estimation de la variance de l'estimateur de la valeur moyenne par placette d'un paramètre dendrométrique. L'introduction de la notion de processus permet de tenir compte du support géométrique des données ce qui n'est pas possible dans le cadre de la théorie des sondages. Comme on ne dispose que d'une réalisation de processus, l'inférence statistique n'est possible qu'avec les hypothèses de stationnarité et d'isotropie qui impliquent que le processus se reproduit identiquement à lui-même dans l'espace et dans toutes les directions.

Les liaisons dues au support géométrique se traduisent par une fonction de corrélation ne dépendant que de la distance entre deux placettes. Une fois cette fonction estimée, la variance obtenue ne dépend que de la forme de la forêt et de la configuration géométrique de l'échantillon systématique. L'application pratique de la méthode n'a été tentée que dans le cas d'un échantillon systématique à maille rectangulaire mais il est possible d'étudier d'une manière analogue tout sondage systématique à l'aide d'un motif géométrique différent.

L'estimation obtenue apparaît nettement préférable pour les paramètres fortement corrélés aux estimations habituelles dans le cas d'un dispositif systématique où les placettes observées sont très concentrées le long d'un petit nombre de layons. Ce type de dispositif est utilisé en pratique dans les forêts denses tropicales pour des raisons évidentes d'économie. Dans le cas de dispositifs systématiques à maille carrée, ou peu concentrée, la formule habituelle du sondage aléatoire donne des résultats analogues.

Il faut remarquer que la formule obtenue pour la variance de l'estimateur ne fait pas intervenir la position dans la forêt de l'échantillon observé (rappelons que cette position dépend du résultat du tirage au hasard de la première placette, les autres s'en déduisant de manière systématique).

Ce ne serait pas le cas si, renonçant à effectuer tout sondage, nous décidions a priori de n'observer qu'une partie de la forêt, choisie à notre convenance. Il faudrait alors calculer la variance de la variable aléatoire $\mathrm{Z}=\overline{\mathrm{X}}-\overline{\overline{\mathrm{X}}}$ où $\overline{\bar{X}}$ désigne la moyenne du paramètre étudié sur la partie observée; ce calcul ferait apparaître en plus de $\bar{r}$ et $\overline{\bar{r}}$ (cf. § 243) la corrélation moyenne entre les placettes de la forêt appartenant au domaine observé et les autres placettes.

Reçu pour publication en novembre 1971.

\section{SUMMARY}

APPLICATION OF THE STOCHASTIC PROCESS THEORY FOR ESTIMATION OF THE PRECISION OF A FOREST INVENTORY BY SYSTEMATIC SAMPLING

Estimation of error in systematic devices remains a crucial problem in the application of sampling theory, especially in the case of forest inventories. Some of the numerous recipes which can be found in literature dealing with sampling methods are enumerated, but they are not completely satisfactory. The authors propose a different approach to this problem using stochastic process theory as it has been suggested by Cochran and others. 
Having defined stationary and isotropic stochastic processes and given their main properties and the conditions of their applicability to forest stands, the authors compute an estimate of the variance of the mean of a one-stage systematic device. The estimator of the variance appears to be a function of both the average correlation coefficient between two sampling units in the whole population, $\overline{\bar{\rho}}$, and the average value of the same in the systematic sample, $\bar{\rho}$. As may be seen, this estimator of the variance depends on the geometric design of the sample and of the shape of the correlogramme, which represents the variation of the correlation coefficient $\rho$ with the distance $d$ between two sampling units. Methods are listed for fitting the function $\rho=f(d)$ by $\rho=p e$-ad $+q e^{-b d}$, and for determining an estimate $\overline{\mathrm{r}}$ of the average value of the correlation coefficient in the whole population.

The method is applied in the case of a forest inventory, using the data of a complete enumeration done in a high mixed tropical forest. Roughly speaking, it appears that the more rectangular the spacing of the sampling units, the more reliable is the estimate of the variance given by this method, especially for parameters corresponding to a species with a grouping distribution. In the case of « random » distributions the estimate of the variance does not differ significantly from the one given by the sampling theory assuming units are randomly selected. A short efficiency study shows that elongated, rectangular spacing is in certain cases the most efficient. This is very important for tropical forests where systematic samples with a close spacing of plots within lines and wide spacing between lines appear to be much less expensive than a square layout due to the relatively high cost of clearing the understorey.

\section{ZUSAMMENFASSUNG}

ANWENDUNG DER STICHPROBENTHEORIE ZUR SCHÄTZUNG DER GENAUIGKEIT EINER AN HAND VON SYSTEMATISCHEN STICHPROBEN DURCHGEFÜHRTEN WALDINVENTUR

Die Berechnung des statistischen Fehlers an Hand von systematischen Stichproben, die sehr häufig in der Forstwirtschaft angewendet werden, stellt ein Problem dar, dessen Lösung nicht im engen Rahmen der Stichprobentheorie zu finden ist. Einen Vorschlag Cochrans und anderer folgend, schlagen die Autoren als Lösung vor, die Forstinventur als Verwirklichung eines stochastischen, stationären und isotropischen Vorganges zu betrachten. Diese Methode ermöglicht eine Berechnung der Schwankungen bei systematischen Stichproben auf Grund der durchsnittlichen Korrelation zwischen den beiden Einheiten jedes einzelnen Paares der untersuchten Population und der Stichproben. Die auf diese Weise berechnete Abweichung hängt daher von der Form der Probe und der Form des Korrelogrammes ab, welches die Änderungen des Korrelationskoeffizienzwischen den Werten eines untersuchten Parameters in zwei Einheiten darstellt, auf Grund der Distanz welche sie voneinander trennt. Berechnungsweisen sind angegeben, und die Methode wird benutzt, wobei Daten einer kompletten tropischen Waldinventur verwendet werden. Diese Methode scheint besonders zu befriedigen, wenn die Maschen der Stichprobeneinheiten rechteckig sind und der untersuchte Parameter eine « ansteckende » Verteilung aufweist.

\section{RÉFÉRENCES BIBLIOGRAPHIQUES}

H. Cramer, M. R. Leadbetter. Stationary and related stochastic processes, J. Wiley and sons Inc. ed. New York.

W. G. Cochran. Sampling techniques (chapitre 8) J. Wiley and sons Inc. ed. New York.

W. G. Cochran. Relative accuracy of systematic and stratified random samples for a certain class of populations. Journal paper n ${ }^{\circ} 1341$ of the Iowa Agricultural Experiment Station, Ames - Iowa.

J. Desabie. Théorie et pratique des sondages. Dunod éd. Paris.

B. Matern. Spatial variation. Medd statens Stogforskningsinst.

F. Yates. Méthodes de sondage pour recensements et enquêtes. Masson et Cie. Dunod éd. Paris.

Sur l'inventaire expérimental réalisé par le C.T.F.T. an Cameroun :

J. P. Lanly. Précision d'un inventaire forestier en fonction de certaines de ses caractéristiques. Bois et Forêts des Tropiques $\mathrm{n}^{\circ} 125$. 